\title{
Meiose e viabilidade polínica na família Araceae ${ }^{1}$
}

\author{
Maria Goreti Senna Corrêa², Judith Viégas²,4, João Baptista da Silva ${ }^{3}$, Paula Fernanda Vaz de Ávila², \\ Gustavo Rossato Busato² e Jaqueline Schneider Lemes ${ }^{2}$
}

Recebido em 10/11/2003. Aceito em 10/09/2004

RESUMO - (Meiose e viabilidade polínica na família Araceae). O objetivo deste trabalho foi analisar a microsporogênese e a viabilidade dos grãos de pólen em 17 espécies de aráceas coletadas no Rio Grande do Sul, Brasil. Nove espécies foram analisadas quanto à ocorrência de células mãe de pólen (CMP) normais e anormais nas fases de metáfase, anáfase e telófase, tanto da meiose I (M I) como da meiose II (M II); 10 espécies foram estudadas quanto à presença de tétrades com número normal ou anormal de micrósporos e 17 espécies quanto à viabilidade dos grãos de pólen. As CMP anormais apresentaram, tanto em M I quanto em M II, cromossomos fora da placa metafásica ou cromossomos retardatários em anáfase e/ou telófase. As freqüências de CMP normais/anormais encontradas na microsporogênese salientam a grande variação existente entre as espécies. Ressalta-se a ausência de CMP com anomalias na microsporogênese de Monstera deliciosa Adans., assim como em M I de Anthurium scandens (Aubl) Engl. e em M II de Caladium hortulanum Birdsey. O número observado de CMP anômalas, em M I e M II, nas espécies Syngonium podophyllum Schott e Zantedeschia aethiopica Spreng, foi maior que o esperado. A freqüência média de tétrades normais em dez espécies de aráceas, assim como a de grãos de pólen viáveis em 17 espécies, foi significativamente superior à freqüência média de anormais e de inviáveis, respectivamente.

Palavras-chave: Araceae, microsporogênese, células-mãe de pólen, comportamento meiótico, viabilidade polínica

\begin{abstract}
Meiosis and pollen viability in Araceae family). The objective of this work was to analyze microsporogenesis and pollen viability in 17 species of the Araceae family collected at Rio Grande do Sul, Brazil. Occurrence of normal and abnormal pollen mother cells (PMC) was analyzed in metaphase, anaphase and telophase, in meiosis I (M I) and meiosis II (M II) of nine species; tetrads with normal or abnormal number of microspores was observed in 10 species, and pollen grain viability, in 17 species. Abnormal PMC presented chromosomes outside the metaphasic plate or laggard chromosomes in anaphase and/or telophase in both M I and M II. The frequencies of normal/abnormal PMC found in microsporogenesis point out to the existence of great variation among the species. It should be emphasized the absence of PMC with abnormalities in Monstera deliciosa Adans. microsporogenesis, as well as in Anthurium scandens (Aubl) Engl. M I and in Caladium hortulanum Birdsey M II. The number of anomalous PMC in Syngonium podophyllum Schott and Zantedeschia aethiopica Spreng in M I and M II was higher than expected. Average frequency of normal tetrads in ten araceous species and viable pollen grains in 17 species were significantly superior to the average frequency of abnormal tetrads and unviable pollen grains, respectively.
\end{abstract}

Key words: Araceae, microsporogenesis, pollen mother cells, meiotic behavior, pollen viability

\section{Introdução}

A família Araceae compreende, com a inclusão das Lemnaceae, cerca de 108 gêneros e 3.750 espécies de Monocotiledôneas herbáceas e trepadeiras. Embora a família ocorra, naturalmente, em todos os continentes, exceto na Antártica, é predominantemente tropical. (IAS 2003). As espécies apresentam grande variabilidade, sendo a maioria constituída de plantas terrestres, mas há várias epífitas e um gênero aquático (Pistia). Enquanto existem aráceas altamente tóxicas, tais como várias espécies de Dieffenbachia, outras são comestíveis, como Colocasia esculenta (L.) Schott e Xanthosoma violaceum Shott. Monstera deliciosa Adans, além de ser bastante usada como ornamental, produz frutos comestíveis. Muitas aráceas são utilizadas como ornamentais, devido à beleza de suas inflorescências e folhas, como por exemplo Anthurium andraeanum Linden, Anthurium scherzerianum Schott, Caladium bicolor (Aiton) Vent., Zantedeschia aethiopica Spreng, Spathiphyllum walisii Regel, Pistia stratiotes L., Syngonium sp., ou medicinais (Acorus calamus, Philodendron selloum) ou, ainda, na produção de

\footnotetext{
1 Parte da Dissertação de Mestrado da primeira Autora

2 Universidade Federal de Pelotas, Instituto de Biologia, Departamento de Zoologia e Genética, Laboratório de Biologia Celular, C. Postal 354, CEP 9601-900, Pelotas, RS, Brasil

3 Universidade Federal de Pelotas, Instituto de Física e Matemática, Departamento de Matemática, Física e Informática

4 Autor para correspondência: juviegas@brturbo.com.br
} 
móveis, cestas e cordas (Heteropsis rigidifolia Engl., Monstera deliciosa, Philodendron selloum). Sendo plantas dos trópicos, as aráceas não se desenvolvem em regiões frias, sendo a temperatura ideal, para seu desenvolvimento, entre $16{ }^{\circ} \mathrm{C}$ e $30^{\circ} \mathrm{C}$, com exceção de Zantedeschia aethiopica Spreng, que suporta temperaturas inferiores a $0{ }^{\circ} \mathrm{C}$ (Reitz 1957; Plantas e Flores 1971; Morton 1987; Leon 1987; Guia Rural 1991; Giacometti \& Leon 1994; Bown 2000).

Segundo Schultz (1990), as aráceas são plantas de caule herbáceo, às vezes lenhoso; curto nas espécies terrestres e palustres, longo e escandente nas epifíticas. Algumas espécies são latescentes e, geralmente, apresentam cristais de oxalato de cálcio nos tecidos. As folhas estão dispostas em rosetas na base ou no ápice do caule, exceto nas espécies escandentes, nas quais são distribuídas de várias maneiras. As folhas são grandes e, freqüentemente, coriáceas. As folhas inteiras ou partidas possuem nervação reticulada ou aberta. Na maioria das espécies terrestres e palustres, as raízes são tuberosas, grandes e ricas em amido; nas ascendentes e epifíticas são adventícias. As flores estão dispostas em uma espiga espessa, a espádice, que é envolvida por uma folha modificada, a espata, freqüentemente de cores vivas. A espata pode ser plana ou, às vezes, imitar um perigônio campanulado, pois uma caldeira fechada, o termóforo, forma-se ao redor da parte basal da espádice. As flores individuais são díclinas (unissexuais) ou monóclinas (hermafroditas), sésseis e muito pequenas. Na espádice de flores díclinas, as flores pistiladas ficam na parte basal e as estaminadas na porção superior. O fruto é sempre uma baga.

A microsporogênese da família Araceae tem sido pouco estudada, apesar do grande número e importância das espécies existentes. Apenas cerca de 100 espécies tiveram parte ou todo processo meiótico estudado, tendo seus números cromossômicos haplóides determinados (Sharma \& Das 1954; Mookerjea 1955; Earl 1957; Pfitzer 1957; Ramachandran 1978; Kaneko \& Kamemoto 1978; Marutani \& Kamemoto 1983; Marutani et al. 1988; 1993; M.G.S. Corrêa, dados não publicados).

Na América do Norte, Japão e Europa desenvolveram-se vários estudos taxonômicos e citogenéticos com os gêneros da família Araceae (F.C. Ramalho, dados não publicados), porém, no Brasil, estes estudos são escassos (F.C. Ramalho, dados não publicados; M.G.S. Corrêa, dados não publicados; Cotias-deOliveira et al. 1999). Visando contribuir com novos conhecimentos sobre a microsporogênese (androspo- rogênese) em Araceae ocorrentes no sul do Brasil e, com isto, proporcionar subsídios para o estudo taxonômico e para trabalhos de melhoramento genético de ornamentais, o presente estudo objetivou realizar análise meiótica em espécies passíveis de se obter inflorescências.

\section{Material e métodos}

Foram estudadas 17 espécies de aráceas coletadas em matas e jardins do Rio Grande do Sul ou adquiridas em floriculturas na cidade de Pelotas, RS, das quais, dependendo do material obtido, foram analisadas as fases da meiose, os tipos de tétrades e/ou a viabilidade dos grãos de pólen (Tab. 1), utilizando-se técnicas convencionais de citogenética, descritas em Guerra \& Souza (2002) e Singh (2002).

Para o estudo das fases da meiose, assim como da viabilidade dos grãos de pólen, as espádices foram coletadas em vários estádios de desenvolvimento, portanto, com comprimentos diversos. As espádices, cortadas transversalmente em pequenas fatias (cerca de $0,5 \mathrm{~cm}$ espes.) ou inteiras, foram fixadas em álcool acético 3:1, por 5 a 24 horas, e estocadas em álcool $70 \%$ até sua utilização. As anteras, retiradas das espádices, foram amaciadas em solução enzimática (celulase $2 \%$ e pectinase 20\%) e esmagadas em uma gota de corante constituída de carmim acético (2\%) ou carmim propiônico (2\%). Este material foi coberto com lamínula, aquecido brevemente sobre chama de lamparina, prensado manualmente e lutado com cola de borracha.

Espádices de Zantedeschia aethiopica foram utilizadas como modelo para otimização do amaciamento das paredes das células-mãe de pólen (CMP) e da obtenção de melhor contraste entre o material cromossômico e a massa citoplasmática das CMP. Para verificar o melhor tempo de incubação na solução enzimática, para o amaciamento das paredes das CMP, as anteras de uma flor foram colocadas sobre lâmina, cobertas com $0,025 \mathrm{~mL}$ de solução enzimática e incubadas em câmara úmida, a $37 \pm 1{ }^{\circ} \mathrm{C}$, pelos períodos de 30, 45 e 60 minutos. Para a obtenção de melhor contraste entre cromossomos e citoplasma, foram avaliadas diferentes concentrações dos corantes carmim acético e carmim propiônico (0,6; 1,0 e 2,0\%) e a adição ou não de hidrato de cloral $(0,025 \mathrm{~mL}$ de hidrato de cloral: $2 \mathrm{~mL}$ do corante), conforme Galli et al. (1998). Para o preparo das lâminas de grãos de pólen, não foi realizado o tratamento enzimático e o corante utilizado foi o carmim acético $2 \%$. 
Tabela 1. Fases da microsporogênese estudadas em 17 espécies de Araceae coletadas no Rio Grande do Sul. P = prófase, M = metáfase, $\mathrm{A}=$ anáfase, $\mathrm{T}=$ telófase, $\mathrm{Te}=$ tétrades.

\begin{tabular}{|c|c|c|c|c|c|c|c|c|c|c|}
\hline \multirow[t]{2}{*}{ Espécies } & \multicolumn{4}{|c|}{ Meiose I } & \multicolumn{4}{|c|}{ Meiose II } & \multirow[t]{2}{*}{$\mathrm{Te}$} & \multirow[t]{2}{*}{ Pólen } \\
\hline & $\mathrm{P}$ & $\mathrm{M}$ & A & $\mathrm{T}$ & $\mathrm{P}$ & $\mathrm{M}$ & A & $\mathrm{T}$ & & \\
\hline Anthurium andreanum Linden * & $\mathrm{X}$ & $\mathrm{X}$ & $\mathrm{X}$ & $\mathrm{X}$ & $\mathrm{X}$ & $\mathrm{X}$ & $\mathrm{X}$ & $\mathrm{X}$ & $\mathrm{X}$ & $\mathrm{X}$ \\
\hline Anthurium gaudichaudianum Kunth & & & & & & & & & & $\mathrm{X}$ \\
\hline Anthurium scandens (Aubl) Engl. & $\mathrm{X}$ & $\mathrm{X}$ & $\mathrm{X}$ & $\mathrm{X}$ & $\mathrm{X}$ & $\mathrm{X}$ & $\mathrm{X}$ & $\mathrm{X}$ & $\mathrm{X}$ & $\mathrm{X}$ \\
\hline Anthurium scherzerianum Schott* & & & & & & & & & & $X$ \\
\hline Caladium hortulanum Birdsey * & $\mathrm{X}$ & $\mathrm{X}$ & $\mathrm{X}$ & $\mathrm{X}$ & $\mathrm{X}$ & $\mathrm{X}$ & $\mathrm{X}$ & $\mathrm{X}$ & $\mathrm{X}$ & $\mathrm{X}$ \\
\hline Colocasia sp. Schott * & $\mathrm{X}$ & $\mathrm{X}$ & $\mathrm{X}$ & $\mathrm{X}$ & $\mathrm{X}$ & $\mathrm{X}$ & $\mathrm{X}$ & $\mathrm{X}$ & & $\mathrm{X}$ \\
\hline Dieffenbachia picta Schott* & & & & & & & & & & $\mathrm{X}$ \\
\hline Mangonia tweediana Schott & & & & & & & & & & $\mathrm{X}$ \\
\hline Monstera deliciosa Adans. * & $\mathrm{X}$ & $\mathrm{X}$ & $\mathrm{X}$ & $\mathrm{X}$ & $\mathrm{X}$ & $\mathrm{X}$ & $\mathrm{X}$ & $\mathrm{X}$ & $\mathrm{X}$ & $\mathrm{X}$ \\
\hline Philodendron cf. imbe Schott * & $\mathrm{X}$ & $\mathrm{X}$ & $\mathrm{X}$ & $\mathrm{X}$ & $\mathrm{X}$ & $\mathrm{X}$ & $\mathrm{X}$ & $\mathrm{X}$ & $\mathrm{X}$ & $\mathrm{X}$ \\
\hline Philodendron missionum Hauman & & & & & & & & & & $\mathrm{X}$ \\
\hline Philodendron undulatum Engl. * & $\mathrm{X}$ & & & & & & & & $\mathrm{X}$ & $\mathrm{X}$ \\
\hline Pistia stratiotes L. & & & & & & & & & & $\mathrm{X}$ \\
\hline Spathiphyllum wallisii Regel* & $\mathrm{X}$ & $\mathrm{X}$ & $\mathrm{X}$ & $\mathrm{X}$ & $\mathrm{X}$ & $\mathrm{X}$ & $\mathrm{X}$ & $\mathrm{X}$ & $\mathrm{X}$ & $\mathrm{X}$ \\
\hline Syngonium podophyllum Schott * & $\mathrm{X}$ & $\mathrm{X}$ & $\mathrm{X}$ & $\mathrm{X}$ & & $\mathrm{X}$ & $\mathrm{X}$ & $\mathrm{X}$ & $\mathrm{X}$ & $\mathrm{X}$ \\
\hline Xanthosoma jacquinii Schott* & & & & & & & & & $\mathrm{X}$ & $\mathrm{X}$ \\
\hline Zantedeschia aethiopica Spreng & $\mathrm{X}$ & $\mathrm{X}$ & $\mathrm{X}$ & $\mathrm{X}$ & $\mathrm{X}$ & $\mathrm{X}$ & $\mathrm{X}$ & $\mathrm{X}$ & $\mathrm{X}$ & $\mathrm{X}$ \\
\hline
\end{tabular}

* = espécies cultivadas

As fases de metáfase I e II, anáfase I e II e telófase I e II foram avaliadas em nove espécies (Tab. 1) quanto à ocorrência de células-mãe de pólen normais e anormais. Foram consideradas CMP anormais aquelas com cromossomos fora da placa equatorial, cromossomos retardatários e/ou pontes cromossômicas. A análise estatística do comportamento meiótico foi feita por meio do teste Qui-quadrado $\left(\chi^{2}\right)$ de independência entre os atributos: espécies (nove espécies) e tipos de células (normais e anormais).

As tétrades foram avaliadas quanto ao número e viabilidade dos micrósporos em dez espécies (Tab. 1), no delineamento inteiramente casualizado, com diferentes números de repetições, que variaram de 4 a 24. As CMP com 4 micrósporos viáveis foram consideradas tétrades normais, as que apresentaram menos de 4 micrósporos (díades e tríades), mais de 4 micrósporos (políades) ou tétrades com micrósporos estéreis (não corados) e/ou com microcélulas foram consideradas anormais.

Os grãos de pólen das 17 espécies (Tab. 1) foram avaliados quanto aos fatores espécie e viabilidade do pólen, em experimento inteiramente casualizado, com três repetições. Para cada espécie, analisaram-se 200 grãos de pólen por repetição. A variável resposta, número dos grãos de pólen viáveis e inviáveis foi transformada por $\sqrt{\mathrm{x}+1}$, antes da análise da variação. As médias foram comparadas pelo teste de Duncan, ao nível de 5\%. Foram considerados inviáveis os grãos de pólen não corados ou com morfologia anômala, ou seja, com tamanhos menores que a maioria, com diminuição da porção citoplasmática ou com parede enrugada.

As análises estatísticas foram realizadas com o auxílio do Sistema de Análises Estatísticas para Microcomputadores/SANEST (Zonta et al. 1984).

\section{Resultados e discussão}

Células-mãe de pólen nos diversos estágios da divisão meiótica foram obtidas em espádices que mediram de 0,5 cm compr. (Spathiphyllum wallisii) a 17,0 cm (Philodendron undulatum), e os grãos de pólen puderam ser analisados, quanto à viabilidade, em espádices de 1,2 cm (Anthurium scherzerianum) a 18,5 cm (Philodendron undulatum), ressaltando a grande variabilidade encontrada entre as espécies estudadas (Tab. 2).

O amaciamento das CMP de Z. aethiopica por incubação, por 30 minutos, na solução enzimática não foi efetivo, apresentando resultados semelhantes aos do preparo de anteras sem o uso de solução enzimática. A incubação por 45 ou 60 minutos promoveu melhores resultados, possibilitando melhor visualização dos cromossomos, tendo sido eleita a de 45 minutos por apresentar resultado mais rápido. Este amaciamento das paredes das CMP por incubação em solução 
Tabela 2. Relação entre os tipos de células encontrados nas anteras e o tamanho da espádice em 17 espécies de Araceae, coletadas no Rio Grande do Sul, Brasil. CMP = células mãe de pólen; Te = tétrades, * = espádices que somente apresentaram prófase I e tétrades.

\begin{tabular}{|c|c|c|c|c|}
\hline \multirow[t]{2}{*}{ Espécies coletadas } & \multirow{2}{*}{$\begin{array}{c}\text { Espádices } \\
\text { compr. (cm) }\end{array}$} & \multicolumn{3}{|c|}{ Tipos de células } \\
\hline & & CMP & $\mathrm{Te}$ & Pólen \\
\hline Anthurium andreanum & $\begin{array}{c}1,3-2,0 \\
5,0\end{array}$ & $\mathrm{X}$ & $\mathrm{X}$ & $\mathrm{X}$ \\
\hline Anthurium gaudichaudianum & 2,8 & & & $\mathrm{X}$ \\
\hline Anthurium scandens & $\begin{array}{l}1,1 \\
1,7\end{array}$ & $\mathrm{X}$ & $\mathrm{X}$ & $\mathrm{X}$ \\
\hline Anthurium scherzerianum & 1,2 & & & $\mathrm{X}$ \\
\hline Caladium hortulanum & $5,5-6,5$ & & & $\mathrm{X}$ \\
\hline Colocasia sp. & $\begin{array}{r}9,0 \\
12,0\end{array}$ & $\mathrm{X}$ & & $\mathrm{X}$ \\
\hline Dieffenbachia picta & 9,0 & & & $\mathrm{X}$ \\
\hline Mangonia tweediana & 3,5 & & & $\mathrm{X}$ \\
\hline Monstera deliciosa & $\begin{array}{r}8,5 \\
10,5\end{array}$ & $X$ & $\mathrm{X}$ & $\mathrm{X}$ \\
\hline Philodendron cf. imbe & $\begin{array}{r}9,5 \\
10,5 \\
14,5\end{array}$ & $\mathrm{X}$ & $\mathrm{X}$ & $\mathrm{X}$ \\
\hline Philodendron missionum & & & & $\mathrm{X}$ \\
\hline Philodendron undulatum & $\begin{array}{c}14,5-17,0 \\
18,5\end{array}$ & $\mathrm{X}^{*}$ & $\mathrm{X}^{*}$ & X \\
\hline Pistia stratiotes** & $0,2-0,5$ & & & $\mathrm{X}$ \\
\hline Spathiphyllum wallisii & $\begin{array}{c}0,5-1,5 \\
2,0\end{array}$ & $\mathrm{X}$ & $\mathrm{X}$ & X \\
\hline Syngonium podophyllum & $\begin{array}{l}4,5 \\
9,5\end{array}$ & $\mathrm{X}$ & $\mathrm{X}$ & X \\
\hline Xanthosoma jacquinii & $\begin{array}{l}11,5 \\
15,0\end{array}$ & & $\mathrm{X}$ & $\mathrm{X}$ \\
\hline Zantedeschia aethiopica & $\begin{array}{l}3,2-4,0 \\
4,5-7,0\end{array}$ & $\mathrm{X}$ & $\mathrm{X}$ & X \\
\hline
\end{tabular}

** = tamanho da flor

enzimática, não é citado nos trabalhos de microsporogênese de aráceas.

Os dois corantes à base de carmim, utilizados em três concentrações diferentes e com ou sem adição de hidrato de cloral (HC), produziram 12 combinações, três das quais propiciaram um contraste regular (carmim propiônico 2\% com ou sem HC e carmim acético 2\% sem HC) e duas, um bom contraste (carmim acético $2 \%$ com ou sem HC); nas demais combinações não houve contraste entre cromossomos e citoplasma. Portanto, foi escolhido o carmim acético 2\% com HC para a coloração das CMP.

Os corantes que têm sido usados para CMP em estudos citológicos de Araceae são a orceína acética 1\% (Kaneko \& Kamemoto 1978; Marutani \& Kamemoto 1983), a orceína acética 2\% (Okada \& Hambali 1989), o carmim acético (Ramachandran 1978;
Sarkar et al. 1979; Chakroborty \& Bhattacharya 1984; Marutani et al. 1988; 1993) e o carmim propiônico (Bai et al. 1971). Nenhum dos pesquisadores citados, no entanto, utilizou a adição de hidrato de cloral para aumentar o contraste entre cromossomos e citoplasma. Esta metodologia foi proposta para CMP de aspargo por Galli et al. (1998) e utilizada em meiose de batata por V. Pandolfi (dados não publicados).

As células mãe de pólen anormais apresentaram cromossomos fora da placa equatorial em metáfase ou cromossomos retardatários em anáfase e telófase (Fig. 1a-b), nas meioses I e II. Não foi verificada a existência de pontes, tanto na meiose I (M I) como na meiose II (M II).

Na Tab. 3, verificam-se as freqüências de CMP normais e anormais encontradas na primeira e na segunda divisão meiótica, salientando-se a grande variação existente entre as nove espécies estudadas. Ressalta-se a ausência de CMP com anormalidades durante o processo meiótico de $M$. deliciosa, assim como em M I de Anthurium scandens e em M II de Caladium hortulanum. Verifica-se, também, a grande presença de CMP anômalas, tanto em M I quanto em M II, das espécies $S$. podophyllum (19,2\% em ambas divisões) e Z. aethiopica (14,0 e 15,5\% em M I e M II, respectivamente).

Houve discrepâncias entre os números observados e esperados de CMP normais e anormais, tanto na primeira como na segunda divisão meiótica das nove espécies analisadas. Conforme Tab. 4, em M I houve grandes diferenças para as espécies Colocasia sp., Philodendron cf. imbe e $M$. deliciosa, nas quais observou-se um número de CMP anormais muito menor do que o esperado. Em M I de S. podophyllum e Z. aethiopica esta relação inverteu-se, ou seja, observou-se um número maior de CMP anormais do que o esperado. De acordo com a Tab. 5, as grandes discrepâncias em M II ficaram por conta de M. deliciosa, onde não foram observadas CMP anormais e de S. podophyllum e Z. aethiopica, para as quais observaram-se números de CMP anormais maiores do que os esperados. Os valores de $\chi^{2}$ foram de 277,33 e 285,43 ( $\mathrm{p}<0,001$ ), altamente significativos, para meiose I e meiose II, respectivamente.

As discrepâncias e a variabilidade interespecífica observadas, no presente estudo, são reforçadas por trabalhos que mostram desde o processo de microsporogênese normal até aquele com a ocorrência de inúmeras irregularidades. Microsporogênese normal foi verificada em Anthurium glaziovii, Anthurium signatum, Zantedeschia elliottiana, Zantedeschia 
albomaculata, Zantedeschia rhemannii e Z. albomaculata $\times Z$. rhemannii e Dieffenbachia picta (Mookerjea 1955; Earl 1957; Ramachandran 1978). Processo meiótico normal, também, foi observado em treze espécies do gênero Anthurium (com exceção de Anthurium ochratum, que apresentou 14 II + 2 I, em diacinese), em seus cultivares e em seus híbridos interespecíficos (Marutani et al. 1988; 1993).

As alterações meióticas encontradas nas espécies Caladium sp., C. bicolor, Dieffenbachia sp., Colocasia antiquorum (diplóide e triplóide), A. andraeanum, A. kamemotoanum, A. roseospadix e Cryptocoryne spiralis incluem cromossomos retardatários e fora da placa equatorial, assim como uma série de outras irregularidades meióticas (Sharma \& Das 1954; Moorkejea 1955; Bai et al. 1971; Ramanchadran 1978; Kaneko \& Kamemoto 1978; Sarkar et al. 1979; Marutani et al. 1993).

A presença de pontes de inversão, além dos cromossomos retardatários, em anáfase I, e a falta de orientação cromossômica na maioria das células em M II foi verificada em $C$. bicolor var. verschafftii (Sharma \& Das 1954). Também, em Caladium bicolor, ocorreram pontes simples e fragmentos acêntricos em 20,0\% das CMP em anáfase I, alterações indicativas de heterozigose para inversão paracêntrica (Ramachandran 1978).

A análise meiótica de Colocasia antiquorum triplóide mostrou pareamento parcial dos cromossomos
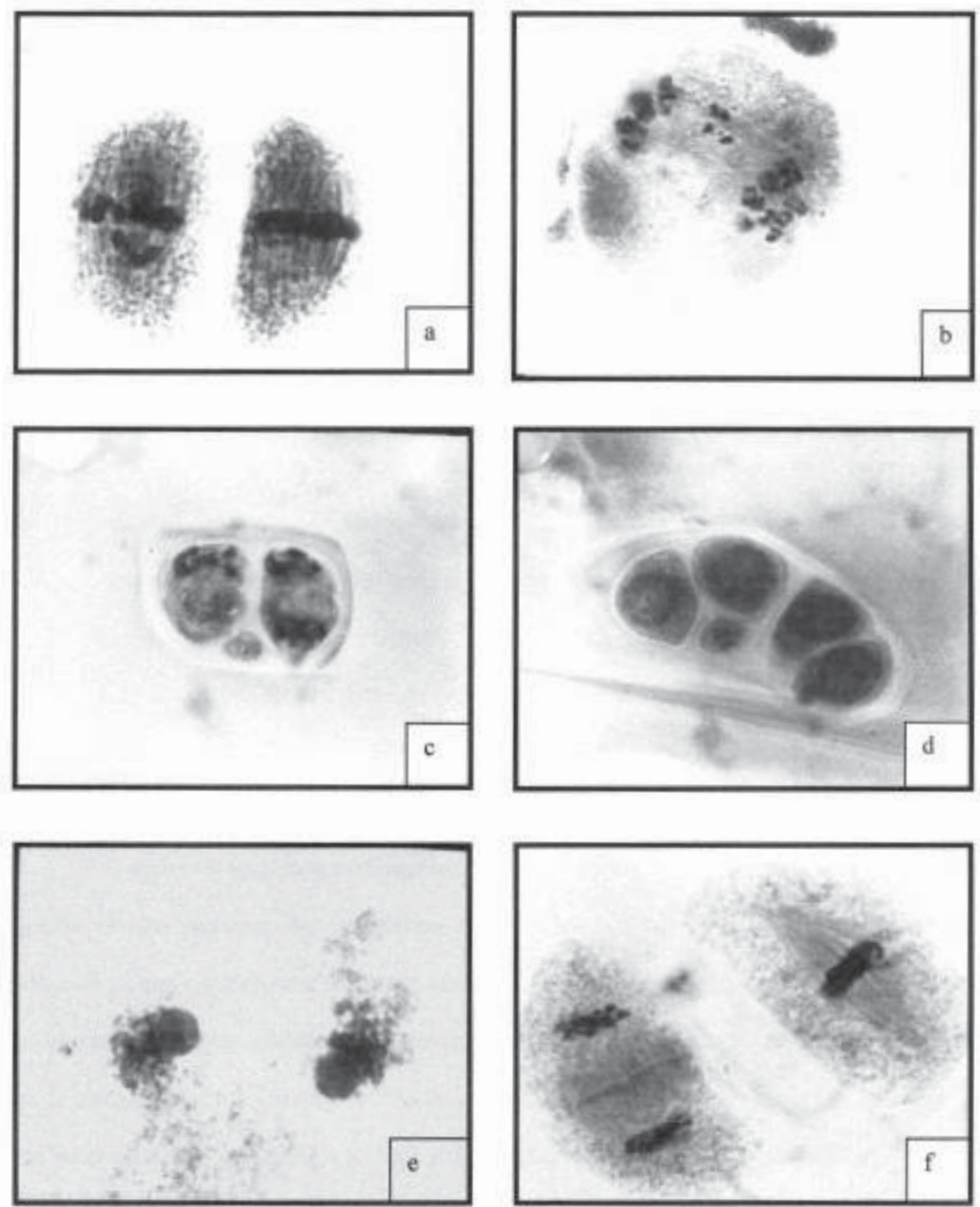

Figura 1. a. Metáfase II com cromossomos fora da placa em Spathiphyllum wallisii Regel; b. Anáfase I com cromossomos retardatários em Zantedeschia aethiopica Spreng: c. Telófase II com microcélula em Anthurium andreanum Linden; d. Tétrade com microcélula em Anthurium andreanum Linden; e. Prófase I com cromossomos em estágio de buquê em Syngonium podophyllum Schott; f. Falta de sincronia em meiose II, díade com metáfase/telófase, em Monstera deliciosa Adans. 
Tabela 3. Freqüências de células-mãe de pólen (CMP) normais e anormais na meiose I e meiose II de nove espécies da família Araceae, coletadas no Rio Grande do Sul, Brasil.

\begin{tabular}{|c|c|c|c|c|c|c|}
\hline \multirow[t]{3}{*}{ Espécies } & \multicolumn{3}{|c|}{ CMP em meiose I } & \multicolumn{3}{|c|}{ CMP em meiose II } \\
\hline & \multirow[t]{2}{*}{ Total } & \multicolumn{2}{|c|}{ Anormais } & \multirow[t]{2}{*}{ Total } & \multicolumn{2}{|c|}{ Anormais } \\
\hline & & $\mathrm{N}^{\mathrm{o}}$ & $\%$ & & $\mathrm{~N}^{\mathrm{o}}$ & $\%$ \\
\hline Anthurium andreanum & 118 & 7 & 5,9 & 188 & 1 & 0,5 \\
\hline Anthurium scandens & 80 & 0 & 0,0 & 33 & 1 & 3,0 \\
\hline Caladium hortulanum & 37 & 2 & 5,4 & 123 & 0 & 0,0 \\
\hline Colocasia sp. & 495 & 9 & 1,8 & 35 & 1 & 2,8 \\
\hline Monstera deliciosa & 794 & 0 & 0,0 & 1182 & 0 & 0,0 \\
\hline Philodendron cf. imbe & 821 & 14 & 1,7 & 80 & 5 & 6,3 \\
\hline Spathiphyllum wallisii & 554 & 55 & 9,9 & 168 & 16 & 9,5 \\
\hline Syngonium podophyllum & 291 & 56 & 19,2 & 546 & 105 & 19,2 \\
\hline Zantedeschia aethiopica & 805 & 113 & 14,0 & 766 & 119 & 15,5 \\
\hline
\end{tabular}

na diacinese e falta de pareamento na M I. As CMP apresentaram, em M I, números variáveis de univalentes, desde 42 até 14 . Foram observadas pontes com fragmentos e cromossomos retardatários nas fases de anáfase e telófase, tanto na M I quanto na M II (Chakraborty \& Bhattacharya 1984). Em Dieffenbachia sp. observou-se a formação de multivalentes e a tendência de agrupamento dos bivalentes (Mookerjea 1955).

Kaneko \& Kamemoto (1978) relataram que as CMP das cv. 'Kaumana' e 'Uniwai' de A. andraeanum apresentaram distúrbio de pareamento em diacinese e $\mathrm{M}$ I, com conseqüente presença de univalentes formadores de retardatários e micronúcleos. Marutani et al. (1993), estudando esses mesmos cultivares, verificaram meiose normal com bom pareamento em M I. Segundo estes últimos autores, as diferenças encontradas devem-se, provavelmente, à observação do pareamento cromossômico em diferentes estágios da meiose e/ou a diferenças nas condições ambientais, relacionadas à temperatura e sazonalidade, que podem afetar o pareamento meiótico. Também, relataram que os cromossomos da cv. 'Kaumana' exibiam adesividade, o que dificultava a análise das configurações meióticas.

As freqüências de CMP com anomalias (Tab. 3) e as discrepâncias entre os números observados e esperados de CMP normais e anormais da M I e M II (Tab. 4 e 5), salientam a regularidade do processo meiótico em $M$. deliciosa e a meiose bastante irregular ocorrente nas espécies $S$. podophyllum e $Z$. aethiopica, além das diferentes freqüências de CMP anormais das outras espécies estudadas (1,7 a 9,9\% em M I e 0,5 a 9,5\% em M II; Tab. 3). O material analisado não apresentou outras alterações, tais como pontes e fragmentos acêntricos. As alterações

Tabela 4. Números observados e esperados, valor de $\chi^{2}$ e sua significância, para células-mãe de pólen (CMP) normais e anormais, na primeira divisão meiótica (meiose I), de nove espécies da família Araceae coletadas no Rio Grande do Sul, Brasil. fo = freqüência observada; fe = freqüência esperada.

\begin{tabular}{|c|c|c|c|c|c|c|}
\hline \multirow[t]{2}{*}{ Espécies } & \multicolumn{3}{|c|}{ CMP normais } & \multicolumn{3}{|c|}{ CMP anormais } \\
\hline & fo & fe & $(\mathrm{fo}-\mathrm{fe})^{2} / \mathrm{fe}$ & fo & fe & $(\text { fo-fe })^{2} / \mathrm{fe}$ \\
\hline Anthurium andraeanum & 111 & 110,4 & 0,00 & 7 & 7,6 & 0,04 \\
\hline Anthurium scandens & 80 & 74,9 & 0,34 & 0 & 5,1 & 5,10 \\
\hline Caladium hortulanum & 35 & 34,6 & 0,00 & 2 & 2,4 & 0,06 \\
\hline Colocasia sp. & 486 & 463,3 & 1,11 & 9 & 31,7 & 16,25 \\
\hline Monstera deliciosa & 794 & 743,1 & 3,48 & 0 & 50,9 & 50,90 \\
\hline Philodendron cf. imbe & 807 & 768,4 & 1,93 & 14 & 52,60 & 28,32 \\
\hline Spathiphyllum wallisii & 499 & 518,5 & 0,73 & 55 & 35,5 & 10,71 \\
\hline Syngonium podophyllum & 235 & 272,3 & 5,10 & 56 & 18,6 & 75,20 \\
\hline Zantedeschia aethiopica & 692 & 753,4 & 5,00 & 113 & 51,6 & 73,06 \\
\hline
\end{tabular}

$\chi^{2}=277,33, \mathrm{p}<0,001$ 
Tabela 5. Números observados e esperados, valor de $\chi^{2}$ e sua significância, de células mãe de pólen (CMP) normais e anormais, na segunda divisão meiótica (meiose II), de nove espécies da família Araceae coletadas no Rio Grande do Sul, Brasil. fo = freqüência observada; fe $=$ freqüência esperada

\begin{tabular}{|c|c|c|c|c|c|c|}
\hline \multirow[t]{2}{*}{ Espécies } & \multicolumn{3}{|c|}{ CMP normais } & \multicolumn{3}{|c|}{ CMP anormais } \\
\hline & fo & fe & $(\text { fo-fe })^{2} / \mathrm{fe}$ & fo & fe & $(f o-f e)^{2} / f e$ \\
\hline Colocasia sp. & 34 & 32,2 & 0,10 & 1 & 2,8 & 1,15 \\
\hline Philodendron cf. imbe & 75 & 73,6 & 0,02 & 5 & 6,4 & 0,30 \\
\hline Anthurium scandens & 32 & 30,4 & 0,08 & 1 & 2,6 & 0,98 \\
\hline Monstera deliciosa & 1182 & $1.087,9$ & 8,13 & 0 & 94,1 & 94,10 \\
\hline Anthurium andraeanum & 187 & 173,1 & 1,11 & 1 & 14,9 & 12,96 \\
\hline Syngonium podophyllum & 441 & 502,6 & 7,54 & 105 & 43,4 & 87,43 \\
\hline Caladium hortulanum & 123 & 113,2 & 0,84 & 0 & 9,8 & 9,80 \\
\hline Zantedeschia aethiopica & 647 & 705,1 & 4,78 & 119 & 60,9 & 55,42 \\
\hline Spathiphyllum wallisii & 148 & 150,9 & 0,05 & 16 & 13,1 & 0,64 \\
\hline
\end{tabular}

$\chi^{2}=277,33, \mathrm{p}<0,001$

ocorridas durante o processo de meiose deverão, teoricamente, refletir-se na viabilidade dos grãos de pólen, os quais resultam diretamente dos tipos de micrósporos encontrados nas tétrades.

A espécie $M$. deliciosa caracterizou-se por apresentar CMP, em meiose II, com díades em estágios diferentes, isto é, díades dessimétricas, com suas células em estágios consecutivos como metáfase/ anáfase e anáfase/telófase e, mais raramente, metáfase/telófase (Fig. 1f). Nas espécies $S$. podophyllum e Philodendron undulatum foi possível observar, com extrema clareza, o chamado "estágio em buquê” ou “nó sinezético”, na prófase I (Fig. 1e). Nos trabalhos publicados sobre meiose em aráceas, não há citação de díades dessimétricas, nem de células no "estágio em buquê".

Os resultados da análise da variação indicaram efeitos altamente significativos para os tratamentos (espécies), nos dois delineamentos inteiramente casualizados. As comparações de médias feitas pelo teste de Duncan, ao nível de 5\%, estão expressas na Tab. 6, que apresenta as freqüências de tétrades normais e de grãos de pólen viáveis observadas, respectivamente, em 10 e em 17 espécies das aráceas. Verificaram-se altas percentagens tanto de tétrades normais (93,1 a 100\%) como de grãos de pólen viáveis (81,6 a $100 \%)$. Apesar de A. andraeanum ter apresentado $100 \%$ de tétrades normais, foi a espécie que apresentou a menor viabilidade polínica (81,9\%), diferindo significativamente das demais, com exceção de $S$. podophyllum, que apresentou comportamento semelhante $(97,1 \%$ de tétrades normais e $88,6 \%$ de pólen viável). Em todas as espécies, sem exceção, a média dos grãos de pólen viáveis foi significativamente superior à de inviáveis.
Cabe ressaltar que $Z$. aethiopica e $S$. podophyllum, que apresentaram as maiores freqüências de CMP anormais, produziram 100\% de tétrades normais e grãos de pólen com boa viabilidade (99,5\% e 88,6\%, respectivamente). Postula-se que, provavelmente, ao final da microsporogênese, ocorra processo seletivo que faz com que se encontre, nestas espécies, apenas tétrades normais, apesar da alta freqüência de CMP anormais. As CMP anômalas seriam descartadas, degenerariam ou teriam a meiose

Tabela 6. Freqüências médias de tétrades normais e de grãos de pólen viáveis de espécies de aráceas coletadas na região sul do Rio Grande do Sul, Brasil.

\begin{tabular}{lcc}
\hline Espécies & $\begin{array}{c}\text { Tétrades normais } \\
(\%)\end{array}$ & $\begin{array}{c}\text { Grãos de pólen } \\
\text { viáveis (\%) }\end{array}$ \\
\hline Zantedeschia aethiopica & $100,0 \mathrm{a}$ & $99,5 \mathrm{a}$ \\
Syngonium podophyllum & $100,0 \mathrm{a}$ & $88,6 \mathrm{ab}$ \\
Philodendron cf. imbe & $99,1 \mathrm{ab}$ & $99,5 \mathrm{a}$ \\
Caladium hortulanum & $98,1 \mathrm{ab}$ & $100,0 \mathrm{a}$ \\
Anthurium scandens & $99,1 \mathrm{abc}$ & $100,0 \mathrm{a}$ \\
Anthurium andraeanum & $97,1 \mathrm{abc}$ & $81,9 \mathrm{~b}$ \\
Spathiphyllum wallisii & $95,9 \mathrm{abc}$ & $95,8 \mathrm{a}$ \\
Monstera deliciosa & $95,8 \mathrm{bc}$ & $100,0 \mathrm{a}$ \\
Philodendron undulatum & $94,5 \mathrm{bc}$ & $100,0 \mathrm{a}$ \\
Xanthosoma jacquinii & $93,1 \mathrm{c}$ & $100,0 \mathrm{a}$ \\
Dieffenbachia picta & - & $100,0 \mathrm{a}$ \\
Mangonia tweedieana & - & $100,0 \mathrm{a}$ \\
Colocasia sp. & - & $98,9 \mathrm{a}$ \\
Philodendron missionum & - & $98,7 \mathrm{a}$ \\
Pistia stratiotes & - & $97,8 \mathrm{a}$ \\
Anthurium gaudichaudianum & - & $97,5 \mathrm{a}$ \\
Anthurium scherzerianum & - & $93,3 \mathrm{a}$
\end{tabular}

Médias seguidas de mesma letra, nas colunas, não diferem entre si, pelo teste de Duncan, nível de 5\% 
interrompida antes da fase de tétrade.

Na literatura, os dados encontrados são bastante contrastantes, pois em alguns gêneros ocorre uma grande variabilidade interespecífica, como é o caso das espécies do gênero Anthurium. Marutani et al. (1988) verificaram alta freqüência de tétrades normais em Anthurium amnicola e seus seis híbridos (88 a 99\%), com exceção do híbrido $A$. amnicola $\times$ A. lindenianum, que apresentou freqüência um pouco menor (82\%). Marutani et al. (1993) relataram uma grande variação na percentagem de tétrades normais em 12 espécies de Anthurium (51,5 a 100\%), sendo que $A$. kamemotoanum, $A$. andraeanum cv. 'Kaumana' e $A$. roseospadix apresentaram os percentuais mais baixos: 51,5, 68 e 78\%, respectivamente. A viabilidade polínica de A. amnicola e seus híbridos, estudada por Marutani et al. (1988), foi de $87,5 \%$ de grãos de pólen férteis, estando a viabilidade do pólen dos híbridos numa faixa entre 3,3 a 27,8\%. Em doze espécies do gênero Anthurium e seus cultivares, analisados por Marutani et al. (1993), a presença de pólen viável variou de 31,1 a 95,5\%, sendo que somente $A$. nymphacifolium e $A$. garagaranum apresentaram viabilidade menor que $50,0 \%$ (31,1 e $37,9 \%$, respectivamente) e outras duas espécies, A. lindenianum e A. andraeanum cv. 'Univai', não produziram grãos de pólen, devido à esterilidade estaminal. A redução da fertilidade do pólen dos híbridos de $A$. andraeanum, cruzado com onze espécies aparentadas, sugere divergência genética entre as espécies utilizadas, apesar da formação regular de bivalentes na prófase I. As espécies de Anthurium, estudadas no presente trabalho, apresentaram altas freqüências de tétrades normais (95,8 a 96,5\%) e de pólen viável (81,9 a 100\%), conforme Tab. 6 .

A viabilidade de $98,9 \%$ encontrada, no presente trabalho, para os grãos de pólen de Colocasia sp., é semelhante às encontradas para Colocasia antiquorum (90\%) e Colocasia esculenta diplóide (95\%). A viabilidade de cerca de 33\% de Colocasia esculenta triplóide é esperada devido ao seu nível de ploidia ímpar (Ramanchadran 1978). Já, os quatorze híbridos diplóides, resultantes do cruzamento entre as espécies diplóides Colocasia esculenta e Colocasia gigantea, apresentaram completa esterilidade dos grãos de pólen, devido à falta de pareamento pela ausência de homologia entre estes dois genomas. Um híbrido triplóide, com um genoma de C. gigantea e dois de C. esculenta, apresentou $21 \%$ de pólen normal em função do pareamento dos dois conjuntos cromossômicos de C. esculenta (Okada \& Hambali
1989). Alta freqüência de pólen viável foi encontrada por Ramanchadran (1978) em outras espécies da família Araceae: Lasia espinosa (98\%), Theriophonum minutum (97\%), Legenandra ovata (94\%), Typhonium divaricatum tetraplóide (90\%) e pentaplóide (86\%) e Caladium bicolor (90\%). Para esta última espécie, Ramanchadran (1978) reportou exatamente o contrário do relatado por Sharma \& Das (1954), que foi de 90\% de esterilidade do pólen.

Cabe destacar que, na espécie A. andraeanum, encontraram-se microcélulas na telófase II e nas tétrades (Fig. 1c-d). Micronúcleos, mas não microcélulas, são citados por Kaneko \& Kamemoto (1978) para esta espécie. Nas demais espécies, analisadas no presente trabalho, também não foram observadas microcélulas, nem micronúcleos.

Uma alta percentagem de grãos de pólen viáveis é esperada como resultado de um alto percentual de tétrades normais, as quais refletiriam diretamente um processo meiótico regular. Sharma \& Das (1954) explicaram o alto percentual de esterilidade do grão de pólen como resultado da ocorrência de irregularidades meióticas. Segundo Marutani et al. (1993), a irregularidade dos movimentos cromossômicos conduz à formação de tétrades com micronúcleos, devido ao retardamento de cromossomos na anáfase II. Para Mendes (1994), a presença de tétrades anormais é indicativa de processo meiótico irregular que, conseqüentemente, leva à produção de pólen com diminuição da viabilidade.

Os dados obtidos possibilitaram verificar que, com exceção de S. podophyllum e Z. aethiopica, a microsporogênese das aráceas coletadas na região sul do Rio Grande do Sul é normal. Estas duas espécies, no entanto, apesar das irregularidades meióticas, produzem grãos de pólen normais e viáveis, provavelmente devido a processo seletivo que acontece no sentido de descarte dos produtos anômalos da microsporogênese, antes da produção das tétrades. A seleção contra CMP anormais e a meiose regular justificam a grande freqüência de tétrades normais e a alta viabilidade dos grãos de pólen das aráceas estudadas.

\section{Agradecimentos}

Ao Prof. Dr. João André Jarenkow e ao Prof. Dr. Jorge Waechter (Departamento de Botânica, IB, UFRGS), pela coleta de algumas espécies e identificação; ao CNPq, CAPES e FAPERGS, pelo apoio financeiro. 


\section{Referências bibliográficas}

Bai, V.K.; Magoon, M.L. \& Krishanan, R. 1971. Meiosis and pollen mitosis in diploid and triploid Colocasia antiquorum Schott. Genetica 42: 187-198.

Bown, D. 2000. Aroids: plants of the Arum family. Oregon, Timber Press, Portland.

Chakraborty, B.N. \& Bhattacharya, G.N. 1984. Desynapsis as well as inversion heterozygosity in the natural population of triploid Colocasia antiquorum Shott. Cytologia 49: 739-743.

Cotias-de-Oliveira, A.L.P.; Guedes, M.L.S \& Barreto, E.C. 1999. Chromosome numbers for Anthurium and Philodendron spp. (Araceae) occurring in Bahia, Brazil. Genetics and Molecular Biology 22: 237-242.

Earl, G. 1957. Chromosome numbers and speciation in the genus Zantedeschia. Plant Life 13: 140-146.

Galli, L.; Viégas, J.; Augustin, E. \& Ecjert, M.I. 1998. Meiosis of anther culture regenerants in asparagus (Asparagus officinalis L.). Genetics and Molecular Biology 21: 93-97.

Giacometti, D.C. \& León, J. 1994. Tannia, Yautia (Xanthosoma sagittifolium). In: J.E. Hernándo Bermejo \& J.E. León (eds.). Neglected Crops: 1492 from a different perspective. FAO Plant Production and Protection Series 26: 253-258.

Guerra, M. \& Souza, M.J. 2002. Como observar cromossomos: um guia de técnicas em citogenética vegetal, animal e humana. Ribeirão Preto, FUNPEC-Editora.

Guia Rural, Plantar. 1991. São Paulo, Editora Abril.

IAS, International Aroid Society. 2003. The genera of Araceae. Online. Disponível na Internet: http://www.aroid.org/ genera/index.html\#a. Acesso em 6/9/2003.

Kaneko, K. \& Kamemoto, H. 1978. Cytological studies of "Kaumana" and "Uniwai" Anthurium. Journal of American Society of Sciences 103: 699-701.

León, J. 1987. Botanica de los cultivos tropicales. San Jose de Costa Rica, Instituto Interamericano de Cooperación para la Agricultura-IICA.
Marutani, N. \& Kamemoto, H. 1983. Transmission and significance of B chromosomes in Anthurium waroqueanum. American Journal of Botany 70: 40-46.

Marutani, N.; Wannakrairoj, S. \& Kamemoto, H. 1988. Chromosome studies on Anthurium amnicola and its hybrids. Aroideana 11: 9-14.

Marutani, N.; Sheffer, R.O. \& Kamemoto, H. 1993. Cytological analysis of Anthurium andraeanum (Araceae) its related taxa and their hybrids. American Journal of Botany 80: 93-103.

Mookerjea, A. 1955. Cytology of different species of aroid with a view to trace the basis of their evolution. Caryologia 7: 221-291.

Morton, J. 1987. Cerimam-Monstera deliciosa. Pp. 15-17. In: Fruits of Warm Climates (J.F. Morton \& F.L. Miami, eds.). Online. Disponível na Internet: http://www.hort.purdue.edu/ newcrop/morton/ceriman.html. Acesso em 6/11/2003.

Okada, H. \& Hambali, G.G. 1989. Chromosome behavior in meiosis of the inter-specific hybrids between Colocasia esculenta (L.) Shott and Colocasia gigantea Hook. F. Cytologia 54: 389-393.

Pfizer, P. 1957. Chromosomenzahlen von araceen. Chromosoma 8: 436-446.

Plantas e Flores. 1971. Aráceas. São Paulo, Abril Cultural, Edição semanal, pp. 181-192.

Ramachandran, K. 1978. Cytological studies on South Indian Araceae. Cytologia 43: 289-303.

Reitz, P.R. 1957. Aráceas catarinenses. Sellowia: Anais Botânicos do Herbário Barbosa Rodrigues 8: 19-70.

Sarkar, A.K.; Dutra, N. \& Chatterlee, U. 1979. Chromosome studies in Cryptocoryne (Araceae). Caryologia 32: 1-4.

Schultz, A. 1990. Introdução à Botânica Sistemática. v.2, Porto Alegre, Editora da Universidade.

Sharma, A.K. \& Das, N.K. 1954. Study of karyotypes and their alterations in aroids. Agronomia Lusitana 16: 23-48.

Singh, R.J. 2002. Plant Cytogenetics. Boca Raton, CRC Press.

Zonta, E.P.; Machado, A.D. \& Silveira Junior, P. 1984. Sanest: Sistema de Análise Estatística para Microcomputadores. Pelotas, Registro na Secretária Especial de InformáticaCategoria A., UFPel. 